\title{
Doxorubicin combined with low intensity ultrasound suppresses the growth of oral squamous cell carcinoma in culture and in xenografts
}

\author{
Haixia Fan ${ }^{1,2+}{ }^{1}$, Haixia Li ${ }^{3+}$, Guanyao Liu' ${ }^{4}$, Wei Cong ${ }^{1}$, Hong Zhao ${ }^{1}$, Wenwu Cao ${ }^{5,6}$ and Jinhua Zheng ${ }^{1 *}$
}

\begin{abstract}
Background: Oral squamous cell carcinoma (OSCC) invades surrounding tissues by upregulating matrix metalloproteinases (MMPs) -2 and -9, which causes over-expression of the Hedgehog signaling proteins Shh and Gli-1 and degradation of the extracellular matrix, thereby creating a "highway" for tumor invasion. We explored the potential of low intensity ultrasound (LIUS) and doxorubicin (DOX) to inhibit the formation of this "highway".

Methods: MTT assays were used to examine OSCC cell viability after exposure to LIUS and DOX. The cell morphological changes and ultrastructure were detected by scanning electron microscopy and transmission electron microscopy. Endogenous autophagy-associated proteins were analyzed by immunofluorescent staining and western blotting. Cell migration and invasion abilities were evaluated by Transwell assays. Collagen fiber changes were evaluated by Masson's trichrome staining. Invasion-associated proteins were analyzed by immunohistochemistry and western blotting.
\end{abstract}

Results: LIUS of $1 \mathrm{~W} / \mathrm{cm}^{2}$ increased the in vitro DOX uptake into OSCC by nearly 3-fold in three different cell lines and induced transient autophagic vacuoles on the cell surface. The combination of LIUS and $0.2 \mu \mathrm{g} / \mathrm{ml} \mathrm{DOX}$ inhibited tumor cell viability and invasion, promoted tumor stromal collagen deposition, and prolonged the survival of mice. This combination also down-regulated MMP-2, MMP-9, Shh and Gli-1 in tumor xenografts. Collagen fiber expression was negatively correlated with the expression of these proteins in human OSCC samples.

Conclusions: Our findings suggest that effective low dosages of DOX in combination with LIUS can inhibit cell proliferation, migration and invasion, which might be through MMP-2/9 production mediated by the Hedgehog signaling pathway.

Keywords: Oral squamous cancer cells, Doxorubicin, Ultrasound, MMP-2/9, Hedgehog signaling pathway

\section{Background}

Despite rapid progress in chemotherapies, radiotherapies, and targeted gene therapies that are used in conjunction with the mainstay treatment of surgery [1-4], the prognosis of oral squamous cell carcinoma (OSCC) is still poor. This is due to the extremely aggressive and metastatic nature of oral cancers. Tumor invasion and

\footnotetext{
* Correspondence: jhzheng@ems.hrbmu.edu.cn

${ }^{\dagger}$ Equal contributors

${ }^{1}$ Departmentof Anatomy, Basic Medical Science College, Harbin Medical University, 194 Xuefu Road, Nangang District, Harbin 150081, China

Full list of author information is available at the end of the article
}

metastasis are dynamic and complicated processes that involve the disruption of the basement membrane (BM) by malignant cells, the degradation of the extracellular matrix (ECM) and the invasion of blood and lymphatic vessels [5]. Proteolysis of the BM and ECM by metalloproteinases (MMPs) is essential for local invasion [6]. Additionally, the Hedgehog (Hh) signaling pathway regulates tumor invasion and metastasis, with its mediators Shh and Gli-1 over-expressed in OSCC [7]. Therefore, the inhibition of collagen fiber degradation in the ECM and BM by MMPs and the modulation of upstream 
signaling molecules in the Hh pathway may be keys to controlling clinical tumor invasion and migration in OSCC.

Doxorubicin (DOX), which can obviously inhibit the synthesis of DNA and RNA, is one of the most effective anti-cancer drugs in several types of cancers $[8,9]$. In combination with LIUS, DOX inhibits the activation of the PI3K/Akt pathway in rat brain glioma [9]. As an upstream signaling activator of PI3K/Akt, the Hh signaling pathway plays an important role in tumor cell proliferation, cell migration and metastasis $[10,11]$. Previous research suggests that the $\mathrm{Hh}$ signaling pathway is activated in oral cancer $[12,13]$. Therefore, DOX may have an advantage over conventional OSCC treatments in that it targets Hh signaling. However, the limitations of DOX have become increasingly evident: DOX has been shown to be associated with increased incidence of cardiomyopathy, and drug tolerance limits its usage in cancer treatment $[14,15]$. Moreover, the drug is almost undetectable in tumors and their surrounding areas after intravenous administration of DOX to mice, which indicates that DOX has a very low permeability and diffusion potential [16]. Therefore, an important research goal would be to find a non-invasive approach to improve local drug permeability in OSCC cells.

Extensive investigation of the biological effects of ultrasound on tissues suggests that the use of ultrasound in combination with chemotherapeutic agents or sonosensitizers may provide an important new therapeutic field. Ultrasound provides a non-invasive and effective approach for the delivery of drugs. Some reports indicate that heat generated by ultrasound can increase the sensitivity to chemotherapy, which has been applied in thermal-chemical treatment $[17,18]$. It is worth noting, however, that the thermal effects of ultrasonic wave propagation in liquids also increase blood circulation in local tissues, which can promote tumor cell extravasation into the blood vessels to produce metastasis [19].

Initially, autophagy was thought to be a survival mechanism in stress responses such as nutrient deprivation and hypoxia [20]. Autophagy is necessary to maintain internal environmental stability. Studies have shown that autophagy is a priority response to apoptosis after ultrasound treatment, suggesting that it may be cytoprotective in the setting of ultrasound treatment [21]. In addition, inhibition of autophagy can increase the sensitivity of genetic, chemo-, or radio-therapy treatments, which can help to eradicate tumor cells [21, 22].

In our previous study, we demonstrated that LIUS combined with Scutellarin or 5-FU can destroy tongue cancer cells and hepatocellular carcinoma cells, with no significant effects on normal cells [23, 24]. The major potential mechanism by which the cell membrane permeability is increased is through mechanical force and cavitation, thereby promoting drug uptake by cancer cells. Furthermore, this treatment method does not easily generate heat, limiting the effect on pathological sites and minimizing the damage to the surrounding normal tissues, which increases its clinical research value. Ultrasound combined with low doses of chemotherapy drugs can have equally curative effects and fewer side effects. Therefore, LIUS may represent a promising treatment modality for enhancing the local effectiveness of chemotherapeutic drugs.

In the present study, we assessed the proliferation and activity of human OSCC lines (SAS, HSC-3 and HSC-4) under differing ultrasonic intensities and durations at a given frequency. Then, we defined the relationship between cell membrane morphological changes and intracellular stress responses at a LIUS of $1 \mathrm{~W} / \mathrm{cm}^{2}$ at different incubation times. We also quantitatively assessed the effectiveness of DOX treatment in combination with LIUS by measuring the expression of collagen fibers, MMP-2, MMP-9, and upstream Hh signaling pathway proteins in in vitro and in vivo experiments. Our results provide an experimental basis for better understanding of the mechanism of LIUS in improving DOX efficacy.

\section{Methods \\ Reagents}

3-(4,5-Dimethyl-2-thiazolyl)-2,5-diphenyl-2H-tetrazo-

lium bromide (MTT), 2-(4-amidinophenyl)-6-indolecarbamidine dihydrochloride (DAPI) and DOX (D1515) were purchased from Sigma-Aldrich (St Louis, MO, USA). Rabbit anti-LC3 (12135-1-AP) was purchased from Proteintech (Chicago, IL, USA), rabbit anti-MMP2 (ab37150) and rabbit anti-MMP-9 (ab38898) were purchased from Abcam (Cambridge, MA, USA), rabbit antiShh (bs-1544R), rabbit anti-Gli-1 (bs-1206R) and rabbit anti-Beclin 1 (bs-1353R) were purchased from Beijing Bioss Biosynthesis Biotechnology (Beijing, China), and mouse anti- $\beta$-actin (sc-47,778) was purchased from Santa Cruz Biotechnology (Santa Cruz, CA, USA).

\section{Cell lines and culture}

Human OSCC cell lines SAS, HSC-4, and HSC-3 and normal HT293 cells were purchased from the Human Science Research Resources Bank (Osaka, Japan) and the Cell Bank of Type Culture Collection of the Chinese Academy of Sciences (Shanghai, China). The cells were cultured in RPMI-1640 supplemented with $10 \%$ fetal bovine serum (FBS) and $1 \%$ penicillin-streptomycin in a humidified atmosphere containing $5 \% \mathrm{CO}_{2}$ at $37{ }^{\circ} \mathrm{C}$. Exponentially growing cells were used in the experiments.

\section{Animals and tumor model}

Male, 4-week-old BALB/c nude mice were purchased from SLAC Laboratory Animal Company (Shanghai, China) $(N=24)$. SAS cell suspensions $\left(1 \times 10^{6} \mathrm{cells} / \mathrm{ml}\right)$ 
were subcutaneously injected into the right-side flanks of mice. When the tumor volume reached $100 \mathrm{~mm}^{3}$ in size (about 7 days after inoculation), the tumor-bearing mice were randomly divided into four groups. Tumor diameters were measured with calipers and calculated using the following formula: $V=\pi / 6 \times L \times S^{2}$, where $L$ and $S$ are the long and short diameters of the tumors. All animal protocols were approved by the Laboratory Animal Committee of the Harbin Medical University.

\section{Clinical samples}

Archived paraffin-embedded OSCC and matched adjacent normal tissues were obtained from 74 patients who had undergone surgical excision at the Harbin Medical University Stomatological Hospital between January 2006 and December 2011. Patient clinical characteristics have been described previously [7]. All patients gave informed consent, and the study was approved by the Research Ethics Committee of Harbin Medical University (Harbin, China).

\section{Ultrasonic exposure}

The ultrasonic generator and power amplifier used in this study were designed and manufactured by the Harbin Institute of Technology (Harbin, China). The ultrasonic setup is described in our previous publication [24] (Additional file 1: Figure S1). For in vitro experiments, the sound pressure level distribution was simulated by finite element simulation using COMSOL as shown in Additional file 1: Figure S2A \& S2B. The ultrasonic transducer (diameter: $40 \mathrm{~mm}$; center frequency: $1.1 \mathrm{MHz}$; duty factor: $20 \%$; repetition frequency: $100 \mathrm{~Hz}$ ) was made of a PZT disk attached to a $2.5 \mathrm{~cm}$ thick aluminum block serving as the acoustic transmission medium. The LIUS intensities in the culture plate were also measured using a PT0907110 needle-type hydrophone $(0.2 \mathrm{~cm}$ active element size, $1-10 \mathrm{MHz}$ bandwidth) (Beijing, China). The peak acoustic pressure was $2.6 \mathrm{MPa}$ and the temporal intensity distribution from the center of the transducer along the direction perpendicular to the transducer is shown in Additional file 1: Figure S2a \& S2b. For the in vivo experiments, the sound pressure level distribution is shown in Additional file 1: Figure S2C \& S2D. The ultrasonic transducer (diameter: $30 \mathrm{~mm}$; center frequency: $1.1 \mathrm{MHz}$; duty factor: 20\%; repetition frequency: $100 \mathrm{~Hz}$ ) was attached to a tapered aluminum buffer head with a $5 \mathrm{~mm}$ diameter front surface that was positioned directly in contact with the skin at the tumor site using an acoustic couplant. The temperature increase in the solution was less than $2{ }^{\circ} \mathrm{C}$ in all experiments.

DOX and ultrasonic treatment of cells in vitro and in vivo For both in vitro and in vivo experiments, four treatment groups were used: 1) non-treatment (Control); 2)
DOX; 3) low intensity ultrasound (LIUS); and 4) DOX in combination with ultrasound (DOX + LIUS).

For the in vitro experiments, cells in the DOX and DOX + LIUS groups were incubated with DOX in the dark. For the control and LIUS groups, an equivalent amount of media was added in place of DOX. After $4 \mathrm{~h}$ incubation, the cells in the LIUS and DOX + LIUS groups were exposed to ultrasound $\left(1.1 \mathrm{MHz}, 1 \mathrm{~W} / \mathrm{cm}^{2}\right.$, $20 \%$ duty cycle) for $3 \mathrm{~min}$ in the dark. After the treatment, the cells were collected for further analyses.

For the in vivo experiments, DOX solution was intraperitoneally injected into tumor-bearing mice in the DOX and DOX + LIUS groups at a dosage of $3 \mathrm{mg} / \mathrm{kg}, 2$ times/week. For comparison, 0.9\% normal saline solution was injected into tumor-bearing mice in the control and LIUS groups. Tumors were irradiated by ultrasound (1.1 $\mathrm{MHz}, 1 \mathrm{~W} / \mathrm{cm}^{2}, 20 \%$ duty cycle) for $5 \mathrm{~min}$ in the dark. The treatment was repeated twice a week for 2 weeks. Tumor diameters and mouse body weights in each group were measured for 14 days. For the prognosis of animals $(N=20)$, the mice were observed daily and sacrificed if they lost $15 \%$ of their body weight. The solid tumors removed from different groups were processed for western blotting, immunohistochemistry and Masson's trichrome staining.

\section{Cell viability and apoptosis assay}

The three human OSCC lines were seeded on detachable 96-well plates and subjected to different treatments for the four groups in each cell line. Then, all groups were cultured for $24 \mathrm{~h}$. Cell viability was quantified by the MTT assay.

In the LIUS and DOX + LIUS groups, the apoptotic cells were detected using Hoechst 33,258 (Sigma-Aldrich) according to the manufacturer's instructions. $\mathrm{Nu}$ clei were visualized by fluorescence microscopy (Olympus, BX51, Japan) with an excitation wavelength of $355 \mathrm{~nm}$ and emission wavelength of $465 \mathrm{~nm}$.

\section{Immunofluorescence}

All OSCC cells were fixed in methanol for about $30 \mathrm{~min}$. Then the cells were blocked with 1\% BSA for $20 \mathrm{~min}$ and incubated with primary antibody (LC3: 1:50; Beclin 1: $1: 100)$ at $4{ }^{\circ} \mathrm{C}$ overnight. After rinsing in PBS, the cells were incubated in secondary antibody for $1 \mathrm{~h}$ followed by counterstaining with DAPI. The cells were examined by fluorescence microscopy (Olympus, BX5, USA).

\section{Scanning electron microscopy (SEM)}

After exposure to different intensities of LIUS, cells were fixed in 2.5\% glutaraldehyde in 0.1 M PBS ( $\mathrm{pH} 7.2-7.4)$ for $24 \mathrm{~h}$. The cells were then immobilized in $1 \%$ osmium tetroxide $\left(\mathrm{OsO}_{4}\right)$, washed with $\mathrm{PBS}$, dehydrated with graded alcohol, displaced, and dried at a critical point. A 
thin layer of gold was evaporated onto the surface before observation under a scanning electron microscope (S3400 N, Hitachi, Japan).

\section{Transmission electron microscopy (TEM)}

Cells were fixed in $2.5 \%$ glutaraldehyde overnight. After washing with $\mathrm{PBS}$, the samples were dehydrated with graded alcohol and embedded in Epon 812 for $72 \mathrm{~h}$ at $60^{\circ}$ C. Ultra-thin sections were cut, stained with uranium acetate and lead citrate, and then observed under a transmission electronic microscope (Hitachi, Tokyo, Japan).

\section{Transwell assays}

In vitro migration/invasion activities were assessed using a BD BioCoat ${ }^{\mathrm{TM}}$ Matrigel $^{\mathrm{TM}}$ Invasion Chamber (BD Biosciences, San Jose, CA, USA). Invasion assays were performed with Falcon cell culture inserts containing $8-\mu \mathrm{m}$ pore size polyethylene terephthalate membranes with a thin layer of matrigel-reconstituted basement membrane. The cancer cells were seeded into the upper chamber $\left(2 \times 10^{4}\right.$ cells/well). After $24 \mathrm{~h}$ incubation at $37{ }^{\circ} \mathrm{C}$, non-invading cells were removed from the upper surfaces of the membranes by scrubbing with cottontipped swabs. Invading cells were fixed with methanol, stained with Giemsa and counted under a microscope. In vitro migration assays were performed according to the same procedure but using PET membranes that were not coated with matrigel.

Cell migration and invasion were quantified by counting the number of cells in 10 visual fields on the lower surface of each filter using phase-contrast microscopy.

\section{Western blotting}

Cells and tissues were lysed in RIPA buffer, separated by $10 \%$ SDS-PAGE, and transferred to nitrocellulose membranes. After blocking with 5\% non-fat dried milk, membranes were incubated with primary antibodies (LC3: 1:500; Beclin 1: 1:500; MMP-2: 1:1000; MMP-9: 1:1000; Shh: 1:100; Gli-1: $1: 100)$ at $4{ }^{\circ} \mathrm{C}$ overnight and then incubated with horseradish peroxidase-conjugated secondary antibodies for $1 \mathrm{~h}$. Signals were detected using an enzymatic Chemiluminescence Kit (Pierce, Rochford, IL, USA). $\beta$-actin (1:500) was used as an internal control.

\section{Histologic examination}

Analysis and evaluation of immunohistochemical results were performed as previously described [25]. The concentrations of primary antibody were 1:100 for Shh and Gli-1, and 1:500 for MMP-2 and MMP-9.

Masson's trichrome staining was used to observe deposited collagenous fibers [22, 26]. Collagen fibers stained blue and muscle fibers stained red. The extent of collagenous fiber expression was assessed per unit area.

\section{Intracellular DOX measurement}

The cells of DOX and DOX + LIUS groups were pretreated with $0.2 \mu \mathrm{g} / \mathrm{ml}$ for $3 \mathrm{~h}$ prior to sonication. For observation of cellular uptake of DOX using fluorescent microscopy (Olympus, BX5, USA), the cells were fixed in $4 \%$ paraformaldehyde for $10 \mathrm{~min}$. The cells were then permeated with $0.1 \%$ Triton X-100 for 5 min. For quantification of intracellular DOX concentrations, the cells in 10 randomly selected fields of view (magnification $\times 200$ ) were counted, and the fluorescence intensity index was used as a measure of cell DOX uptake.

\section{Statistical analysis}

Data are expressed as means \pm standard deviations (SD) of three independent experiments. The data were analyzed by one-way ANOVA. Survival analysis was estimated using the Kaplan-Meier method and compared using the log-rank test. The Spearman's rank correlation coefficient test was used to examine correlations between the expression of collagen fibers, MMP-2, MMP9, Shh and Gli-1. Results were considered significant when $p<0.05$.

\section{Results}

\section{Effects of LIUS and DOX on OSCC cell viability}

To examine the effect of different ultrasonic intensities $\left(0.1,0.5,1.0,2.0,4.0 \mathrm{~W} / \mathrm{cm}^{2}\right)$ on OSCC cell viability, we performed sonication followed by MTT assay. The viability rates of the OSCC cells decreased significantly with increasing sonication intensity. The IC50s were $2.332 \mathrm{~W} / \mathrm{cm}^{2}$ for SAS cells, $2.344 \mathrm{~W} / \mathrm{cm}^{2}$ for $\mathrm{HSC}-4$ cells and $2.647 \mathrm{~W} / \mathrm{cm}^{2}$ for HSC-3 cells (Fig. 1a). These results indicate that ultrasound alone can inhibit the growth of OSCC cells in an intensity-dependent manner, though practically no effect was observed for ultrasound intensities below $1.0 \mathrm{~W} / \mathrm{cm}^{2}$.

To examine the effect of various dosages (0.01, 0.05, $0.1,0.2,0.4,0.8,1.5,2.0 \mu \mathrm{g} / \mathrm{ml}$ ) of DOX on cell viability, we measured SAS, HSC-4 and HSC-3 cell viabilities by MTT assay after incubation with DOX for $24 \mathrm{~h}$. The cell viabilities decreased significantly with increasing DOX ( $p<0.01$, versus control) (Fig. 1b). The IC50 values for SAS, HSC-4 and HSC-3 were $1.161 \mu \mathrm{g} / \mathrm{ml}, 0.811 \mu \mathrm{g} / \mathrm{ml}$ and $0.876 \mu \mathrm{g} / \mathrm{ml}$, respectively (Fig. 1b). These results suggest that DOX inhibits the growth of OSCC cell lines in a dose-dependent manner, but that there is practically no effect when the DOX dosage is below $0.2 \mu \mathrm{g} / \mathrm{ml}$.

\section{Effects of ultrasound on the in vitro cell ultrastructure}

Next, we used SEM to detect morphological changes of SAS cells at different incubation times. As shown in Fig. 1c, "pores" appeared on the cell surface after LIUS $\left(1.0 \mathrm{~W} / \mathrm{cm}^{2}\right)$ treatment for different incubation times. Moreover, the "pores" could be observed up to $9 \mathrm{~h}$ after 

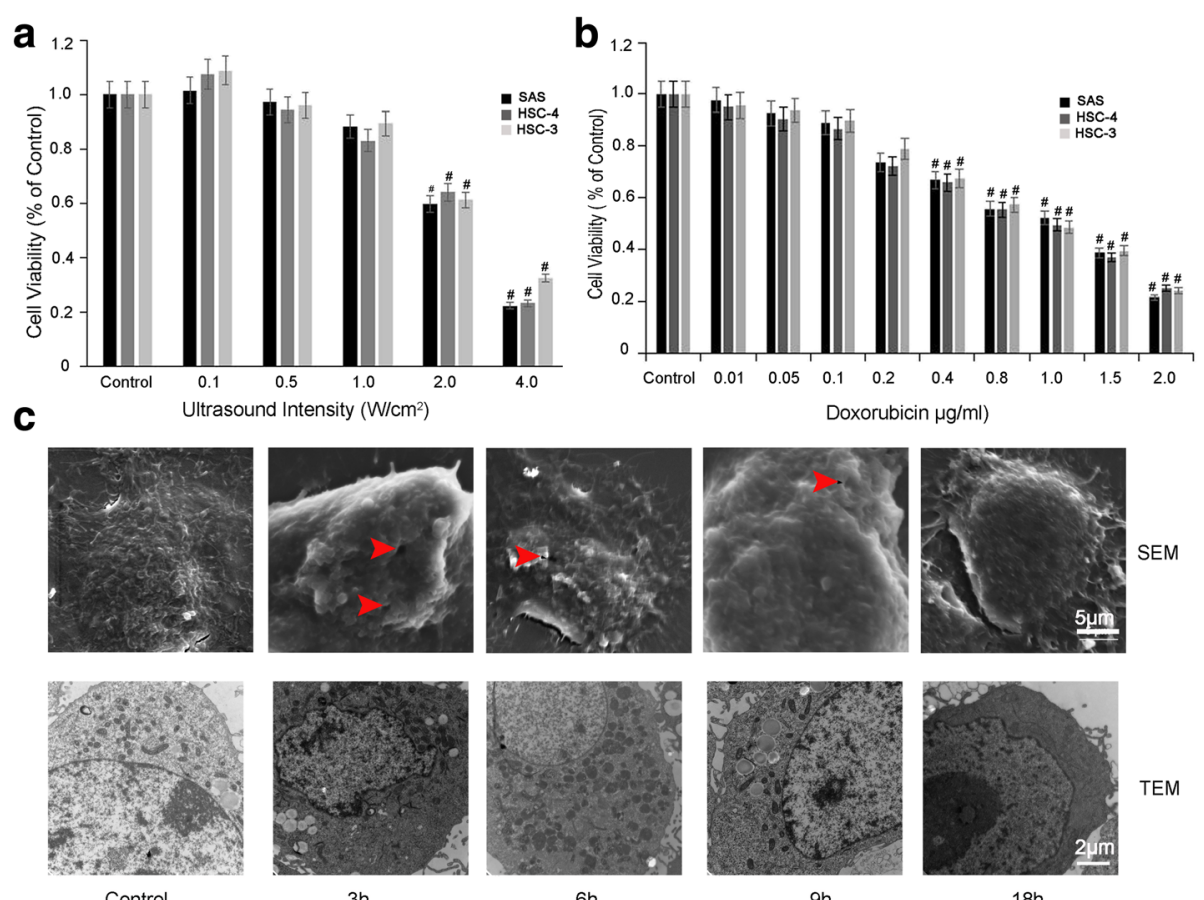

6h

9h

$18 \mathrm{~h}$

Fig. 1 Cytotoxic effects of LIUS and DOX on OSCC cells. Cytotoxic effects were measured by MTT assay after exposure to different ultrasound intensities (a) and different DOX dosage (b) for $24 \mathrm{~h}$. Data are expressed as means \pm SD of three independent experiments. $\mathbf{c}$, The morphological and ultra-structural changes of SAS cancer cells were detected by SEM (upper pane) and TEM (lower panel), at different incubation times after $1 \mathrm{~W} / \mathrm{cm}^{2}$ ultrasound exposure

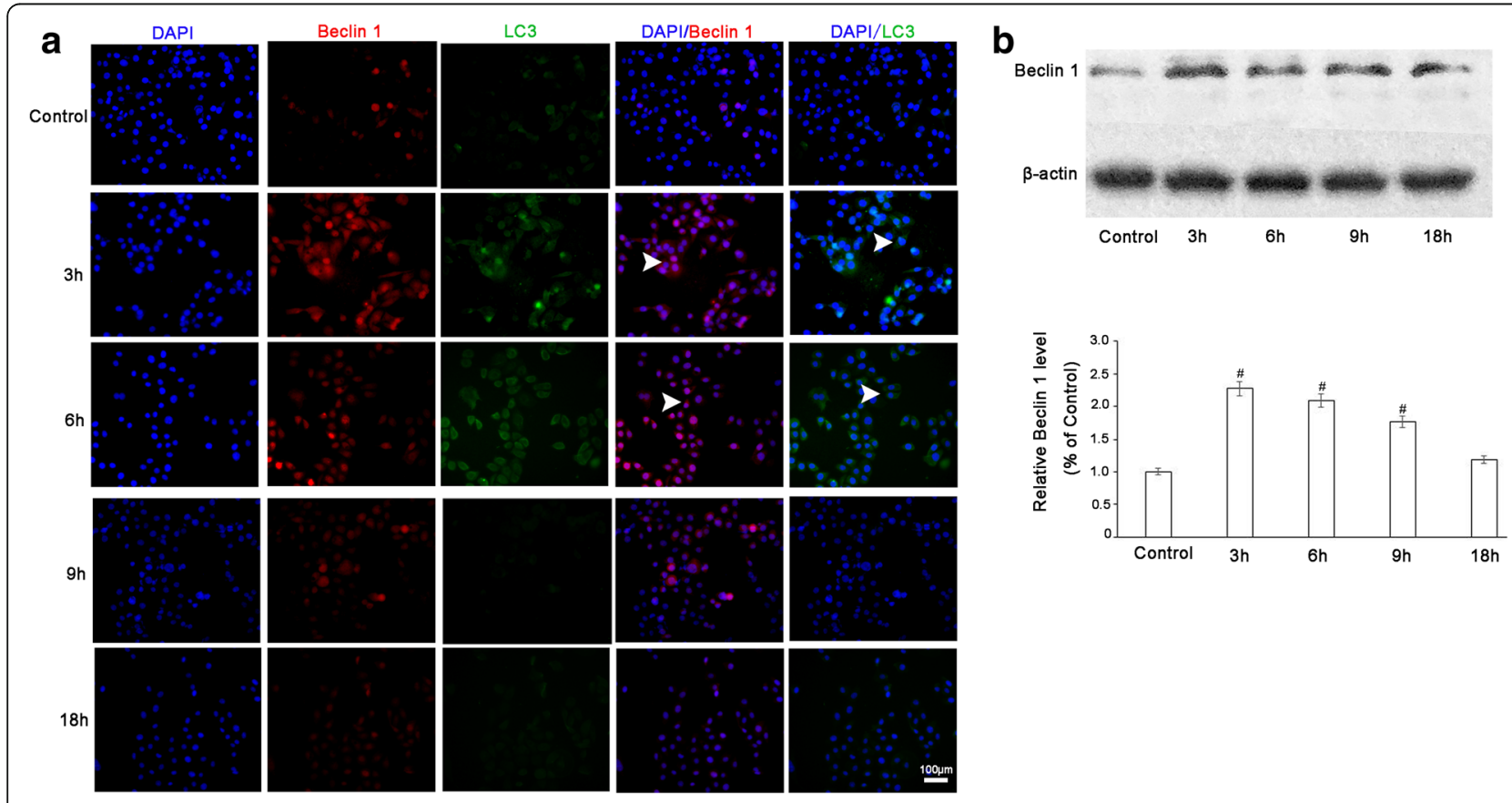

Fig. 2 Expression levels of LC3. Immunofluorescence assay (a) and Western blotting (b) after $1 \mathrm{~W} / \mathrm{cm}^{2}$ LIUS exposure followed by different incubation times. Data are expressed as means \pm SD of three independent experiments. ${ }^{*} p<0.05$ vs. control group, ${ }^{*} p<0.05$ vs. $0 \mathrm{~h}$ 
treatment and disappeared completely at $18 \mathrm{~h}$ after treatment (Fig. 1c, upper panel). These results indicate that $1.0 \mathrm{~W} / \mathrm{cm}^{2}$ ultrasound exposure induced transient "pores" on the cell surface, but that the damage was not permanent and the cells self-recovered upon extended incubation.

To further examine the effects of ultrasound on OSCC, we used TEM to study the ultrastructure of SAS cells after $1.0 \mathrm{~W} / \mathrm{cm}^{2}$ LIUS treatment for different incubation times. As for the SEM, "pores" were visible on the cell membranes, but the membrane structure was still intact, with more cytoplasmic content and clustered nuclear chromatin. At extended incubation times, more cells had autophagic vacuoles containing cell organelles, such as mitochondria and/or endoplasmic reticulum. However, $9 \mathrm{~h}$ after the LIUS treatment, the numbers of autophagic vacuoles began to decrease, and almost no autophagic vacuoles could be detected after $18 \mathrm{~h}$ (Fig. 1c, lower panel). These findings indicate that pore formation and autophagic vacuoles induced by LIUS at $1.0 \mathrm{~W} / \mathrm{cm}^{2}$ were recoverable.

To further verify these results, we examined the expression of the autophagy markers Beclin1 and LC3 by immunofluorescent microscopy. As shown in Fig. 2a, no expression of Beclin 1 and LC3 was detected in the control group. However, cells treated with LIUS showed increased levels of Beclin 1 and LC3 expression, which subsequently decreased at certain times post-treatment. Western blotting further confirmed the changes in Beclin 1 (Fig. 2b).

\section{In vitro and in vivo cytotoxicity of combined DOX and LIUS therapy}

We further investigated whether "pores" can facilitate the entry of DOX as an extracellular chemotherapeutic drug into cells by examining the intracellular DOX accumulation after LIUS $\left(1.0 \mathrm{~W} / \mathrm{cm}^{2}\right)$ and low dosage DOX $(0.2 \mu \mathrm{g} /$ $\mathrm{ml}$ ) combination treatment. As shown in Fig. 3a, DOX was successfully transported into OSCC cells after LIUS treatment (white arrow). Compared to DOX alone, LIUS enhanced DOX uptake by 2.89-fold in SAS cells, 2.69-fold in HSC-4 cells, and 3.23-fold in HSC-3 cells (Fig. 3b). These findings indicate that LIUS exposure dramatically increases the local DOX uptake into OSCC cells.

To examine whether increased DOX entry correlates with enhanced cytotoxic effects, we performed MTT assays $24 \mathrm{~h}$ after DOX and LIUS combination treatment.

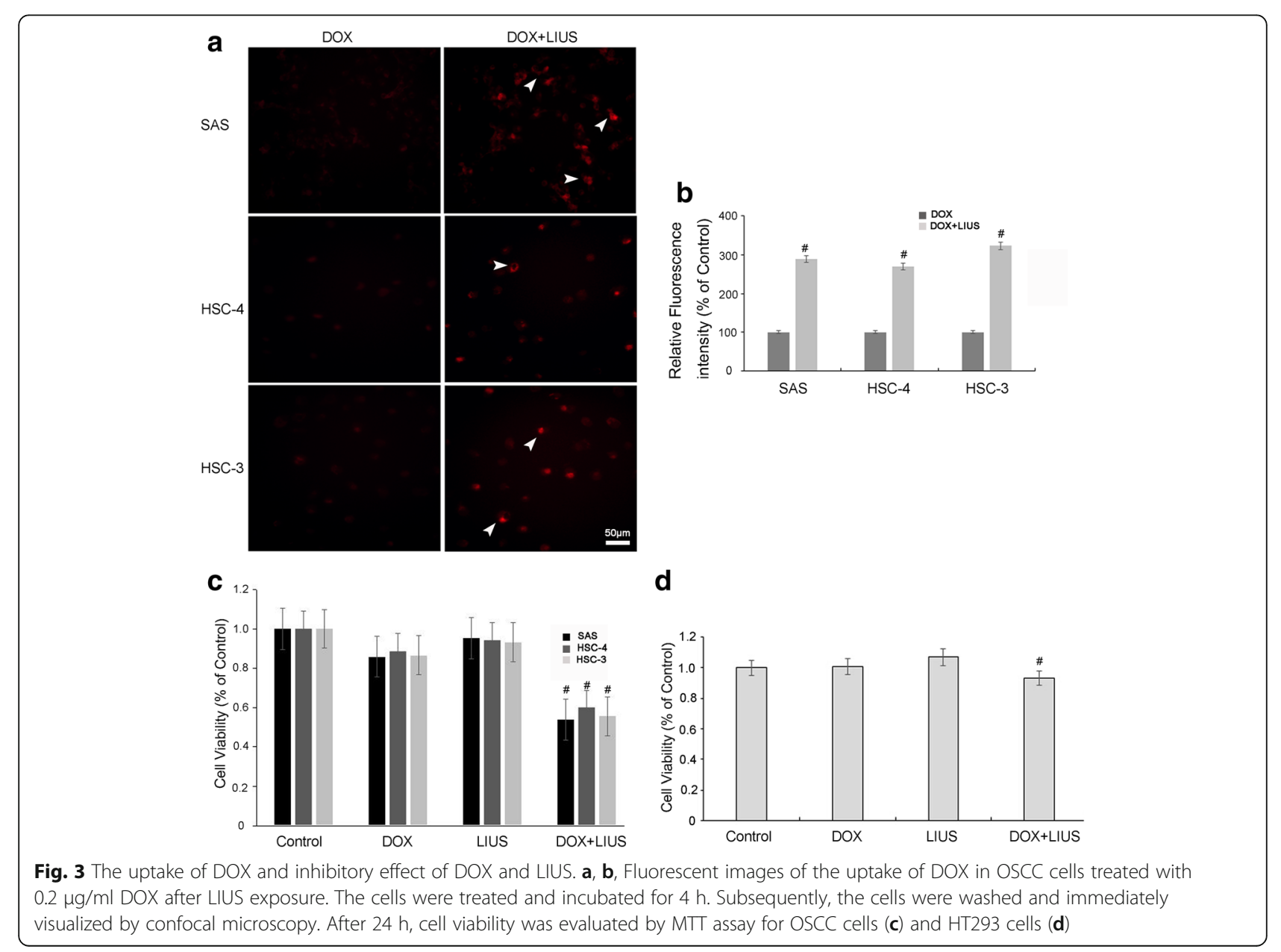


As shown in Fig. 3c, the OSCC cells treated with DOX in combination with LIUS showed significantly decreased cell viability as compared to control cells and cells treated with DOX alone or LIUS alone. Moreover, DOX and LIUS combination treatment did not induce significant cytotoxicity towards non-cancerous HT293 cells (Fig. 3d, and Additional file 1: Figure S3), suggesting that the effects are specific for OSCC cells.

As additional verification of the cytotoxic effects of combination treatment of OSCC cells, we examined the nuclei using Hoechst 33,258 staining after combined treatment (Fig. 4a). Compared to the control, DOX alone and LIUS alone groups, the DOX + LIUS group showed an obvious increase in apoptosis for all three cell lines $(P<0.05$, Fig. $4 \mathrm{a}$ and $\mathrm{b})$. These results indicate that DOX in combination with LIUS significantly induces cell apoptosis in cultured OSCC cells.

To evaluate the in vivo biological effects of DOX in combination with LIUS, we employed a SAS tumor xenograft model in nude BALB/c mice. Compared with the control group, mice treated with DOX alone and
LIUS alone had no obvious tumor growth suppression, while the DOX + LIUS group showed a significant antitumor effect. At the end of the 14-d treatment period, the tumor volume inhibition ratios were $13 \%$ in the DOX group, 7\% in the LIUS group and 55\% in the DOX + LIUS group (Fig. 4c). We also observed a significant increase in the survival rate of mice bearing SAS cells for DOX and LIUS combination treatment compared with DOX alone or LIUS alone treatment $(P<0.05$, Fig. 4d). Furthermore, no adverse effects, such as skin ulceration or toxic death, were observed in any of the groups.

\section{Effects of in vitro DOX and LIUS combination treatment} on tumor cell migration and invasion

We further examined whether DOX in combination with LIUS can inhibit OSCC cell migration and invasion by Transwell assay. Compared to the control group, the LIUS alone and DOX alone groups showed minimal effects on the migration and invasion of OSCC cells. However, the DOX + LIUS group showed significant

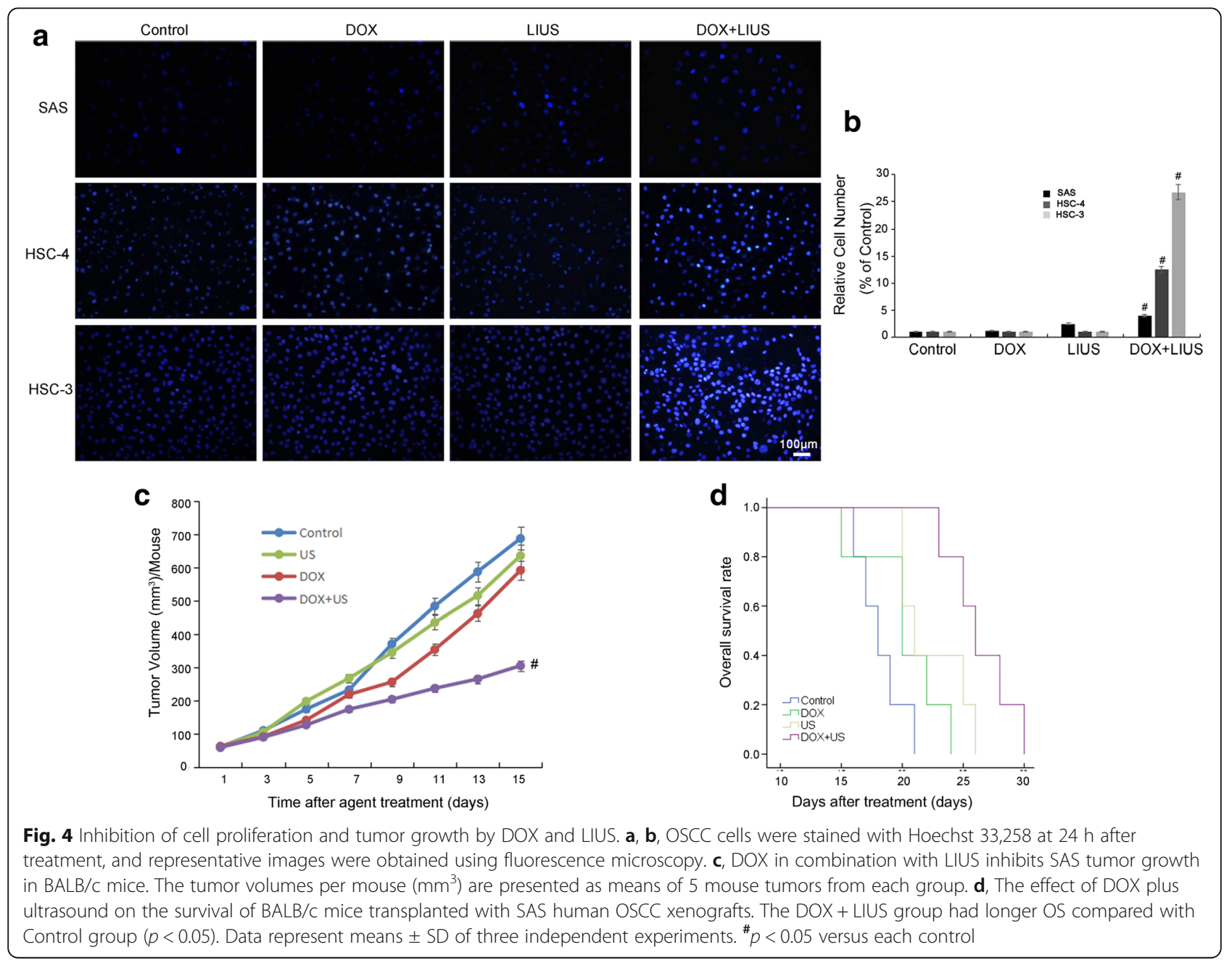


reduction of cell migration (Fig. 5a and b) and invasion (Fig. 5c and d).

To investigate the molecular mechanism of DOX and LIUS combination treatment on cell migration and invasion, we examined effects on signaling molecules that are known to mediate invasiveness, including the Hh signaling pathway mediators Shh and Gli-1 and MMP-2/9. Western blotting assays demonstrated that DOX alone and LIUS alone slightly inhibited the expression of these proteins in OSCC cells. However, DOX in combination with LIUS significantly enhanced inhibition (Fig. $6 a$ and b for SAS cells; Fig. $6 \mathrm{c}$ and d for HSC-4 cells; Figs $6 \mathrm{e}$ and $\mathrm{f}$ for HSC3 cells). These results are consistent with the possibility that inhibition of tumor migration and invasion by DOX in combination with LIUS may be through the downregulation of MMP-2, MMP-9, Shh and Gli-1 expression.

\section{Effects of in vivo DOX and LIUS combination treatment} on the expression of collagen, MMP-2/9, Shh and Gli-1 To determine whether DOX and LIUS combination treatment also reduces the in vivo invasiveness of
OSCC, we assessed the effects on SAS tumor xenograft growth in nude BALB/c mice. Our results demonstrate that collagen fibers in the control, DOX alone and LIUS alone groups appeared thin and disrupted, with a lack of fiber cross-linking and a disordered wavy arrangement. However, collagen fibers in the DOX + LIUS group appeared as dense bundles that penetrated into the tumor stroma (Fig. 7a and b). Because collagen fiber is a major component of the BM, these results are consistent with the decreased invasiveness of OSCC cells after treatment with DOX in combination with LIUS .

Next, we assessed the expression of MMP-2, MMP-9, Shh and Gli-1 by immunohistochemical staining (Fig. 7c and d). Expression of all four proteins was significantly down-regulated in the DOX + LIUS group compared with the control group. Western analysis verified these findings (Fig. 7e and f). These results are consistent with the in vitro results suggesting that LIUS may enhance the inhibitory effects of DOX through down-regulation of MMP-2, MMP-9, Shh and Gli-1 expression.

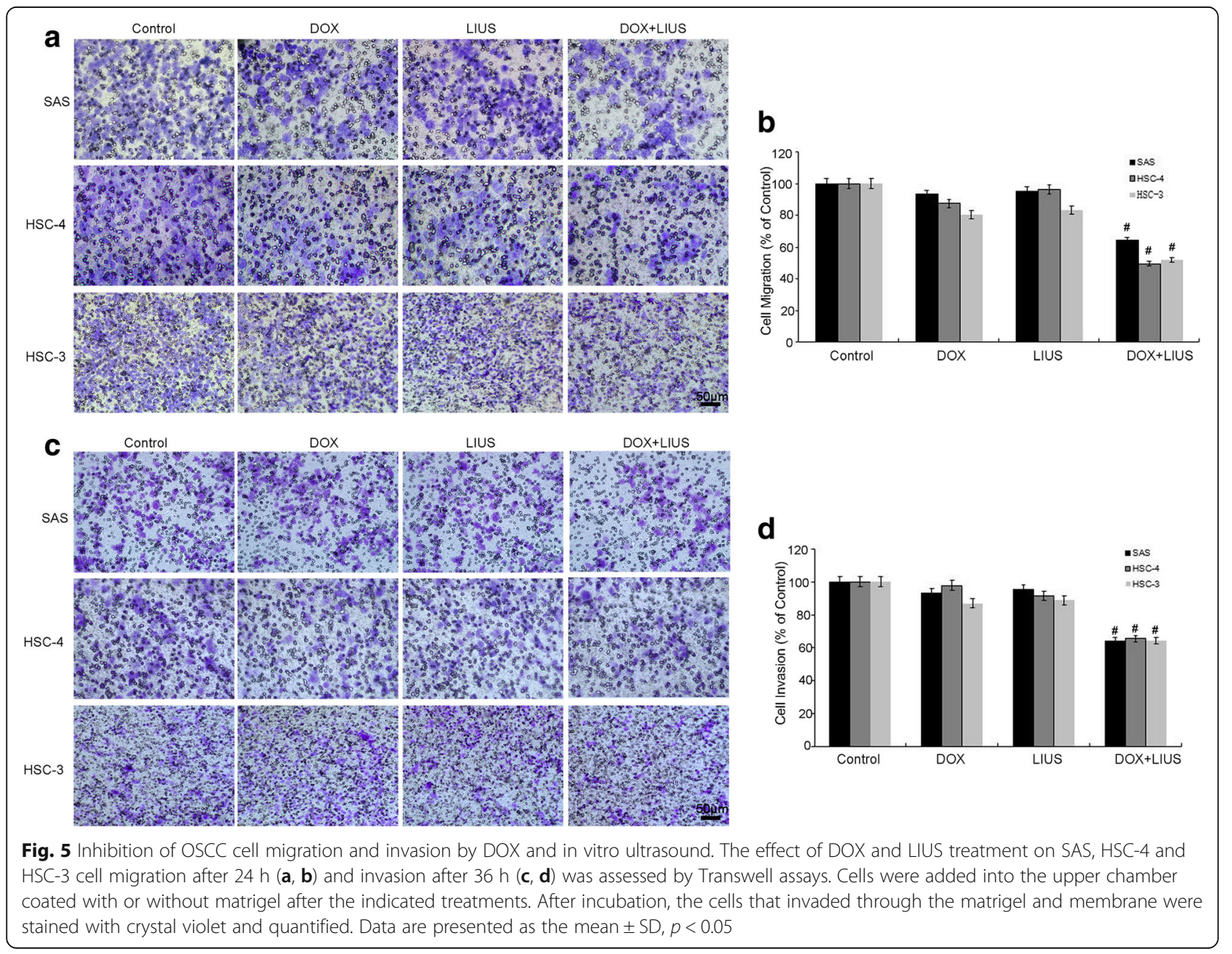



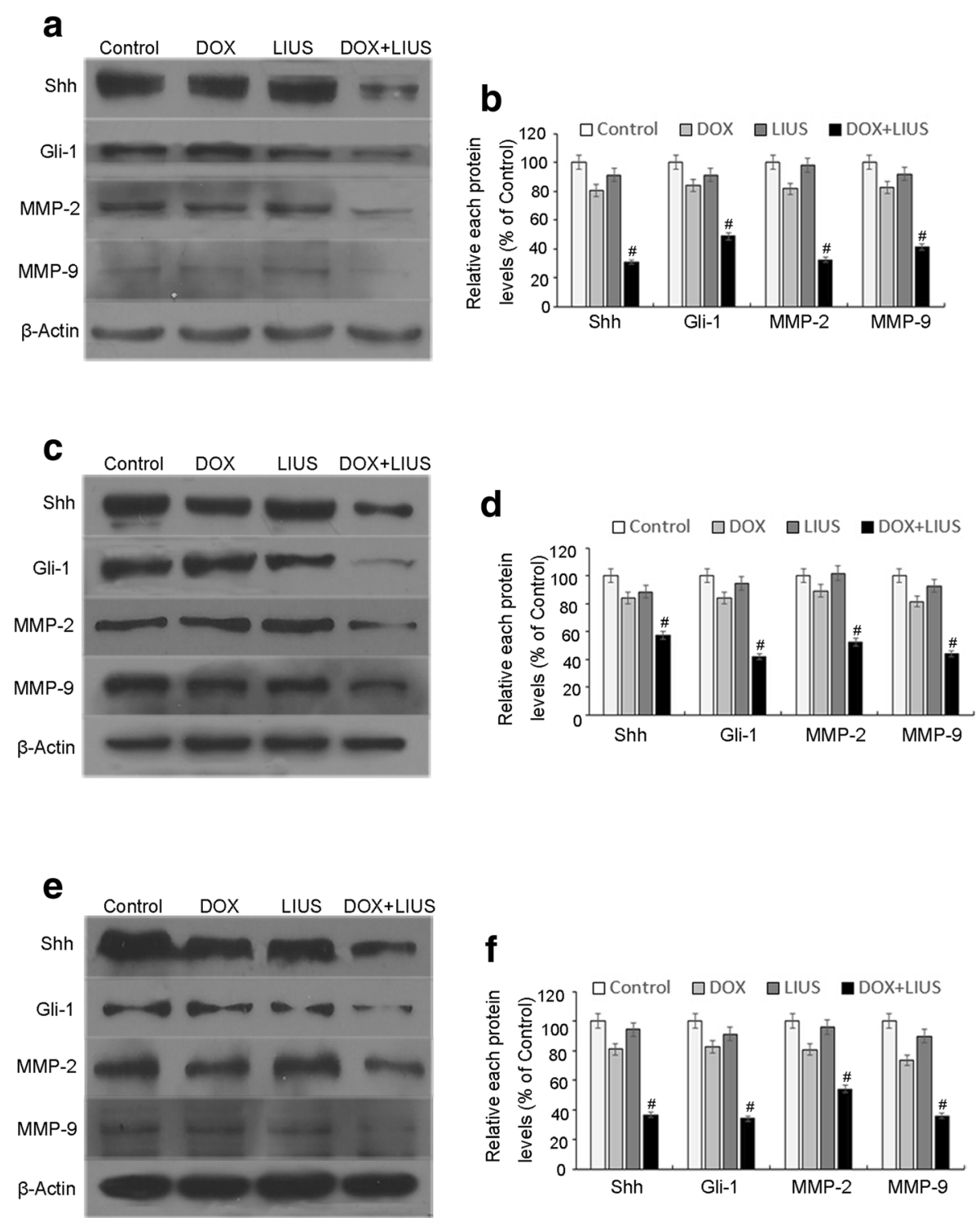

Fig. 6 Expression of Shh, Gli-1, MMP-2 and MMP-9. Expression levels of Shh, Gli-1, MMP-2 and MMP-9 proteins were assessed by Western blotting after treatment of SAS cells $(\mathbf{a}, \mathbf{b})$, HSC-4 cells (c, d) or HSC-3 cells $(\mathbf{e}, \mathbf{f})$. $\beta$-Actin was assessed as an internal control. Representative data from three independent experiments are shown. ${ }^{*} p<0.05$ versus control

\section{Expression of MMP-2/9 and Hh signaling pathway compo- nents in clinical specimens}

In order to evaluate the potential of this combination treatment strategy for chemotherapy of human OSCC patients, it is important to consider whether the targeted tumor markers are over-expressed in primary human OSCC samples. We evaluated the expression of MMP-2, MMP-9, Shh and Gli-1 in 74 pairs of OSCC tissues and adjacent non-cancerous oral tissue samples. In noncancerous tissues, collagen fibers were interwoven into a network, and extensive fiber cross-linking was evidenced by thick bundles (Fig. 8a). By contrast, in OSCC tissues, the collagen fibers were thin and short, with reduced cross-linking and a disordered wavy appearance (Fig. 8f). Furthermore, the immunoreactivity of MMP-2, MMP-9, Shh and Gli-1 was increased in OSCC tissues compared with matched adjacent non-cancerous oral tissues (Figs. 8b-e, g-j). We further investigated the correlation between collagen fibers and the expression of MMP-2, MMP-9 and the Hh pathway components in human OSCC samples by Spearman analysis. As shown in Table 1, collagen fiber expression correlated negatively with the expression of each of these proteins. These results suggest that the expression of MMP-2, MMP-9, Shh, and Gli-1 are up-regulated in human OSCC tissues compared with non-cancerous tissues, which provides a 


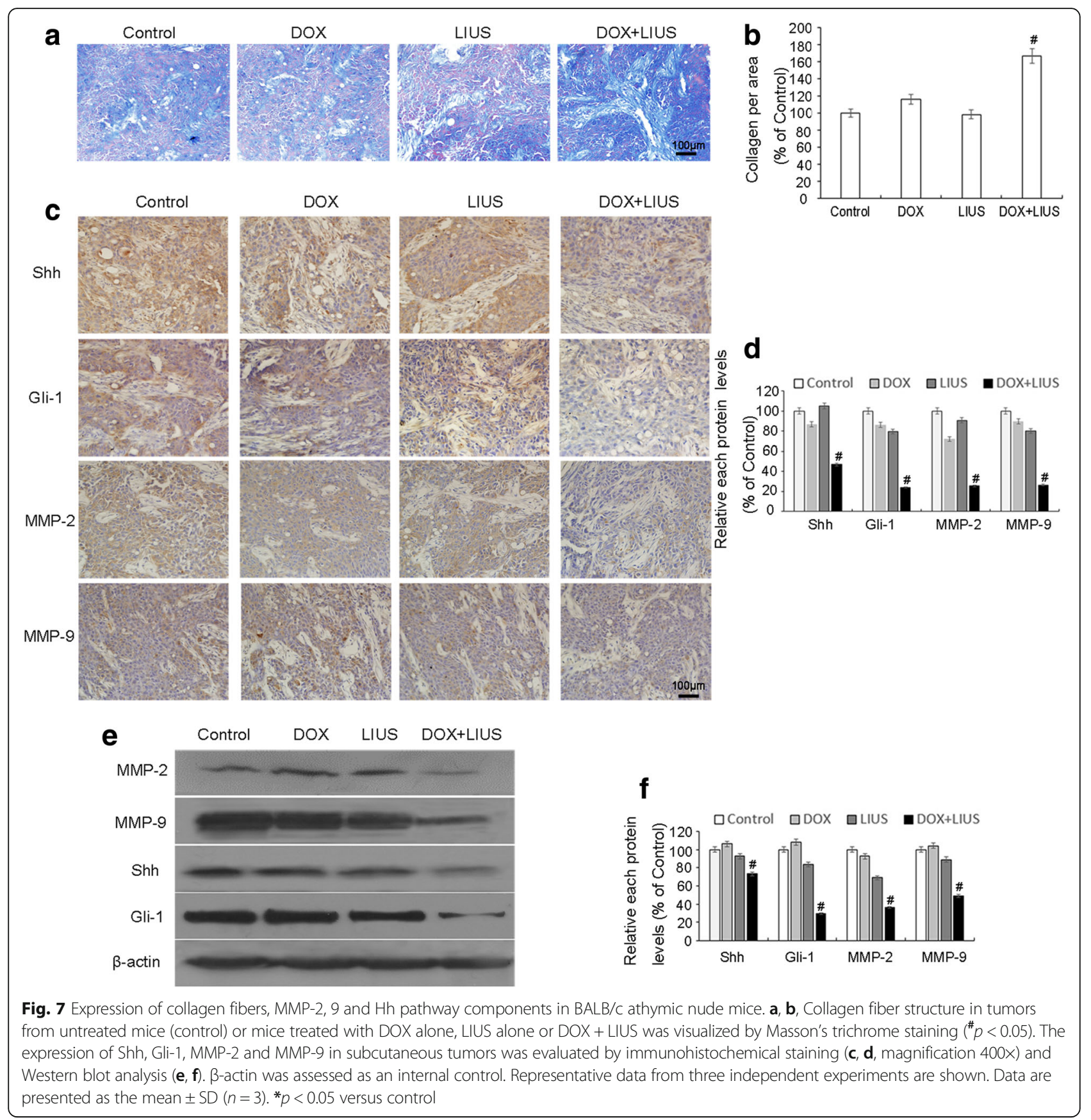

rationale for applying DOX and LIUS in combination in future clinical studies.

\section{Discussion}

Within a certain range of intensity, the proliferation of cancer cells increases significantly with increases in the sonication intensity. However, ultrasound intensities above a certain threshold cause cellular structural and functional changes. Consequently, biological ultrasound induces cell proliferation or changes in cellular structures or functions [27]. By performing MTT assays, we demonstrated that the viability rates of OSCC cells decreases significantly with increasing sonication intensity (ranging from $0.5-4 \mathrm{~W} / \mathrm{cm}^{2}$ ). Compared to the cell viability of the control group, there was no effect on OSCC cell viability when the ultrasound intensity was $1.0 \mathrm{~W} /$ $\mathrm{cm}^{2}$ (Fig. 1a).

Using SEM, we also found that "pores" immediately formed in cell membranes after $1 \mathrm{~W} / \mathrm{cm}^{2}$ LIUS exposure, and that they completely disappeared after $18 \mathrm{~h}$ incubation (Fig. 1c). Therefore, we speculate that the "pores" are self-recoverable. Intracellular autophagic 


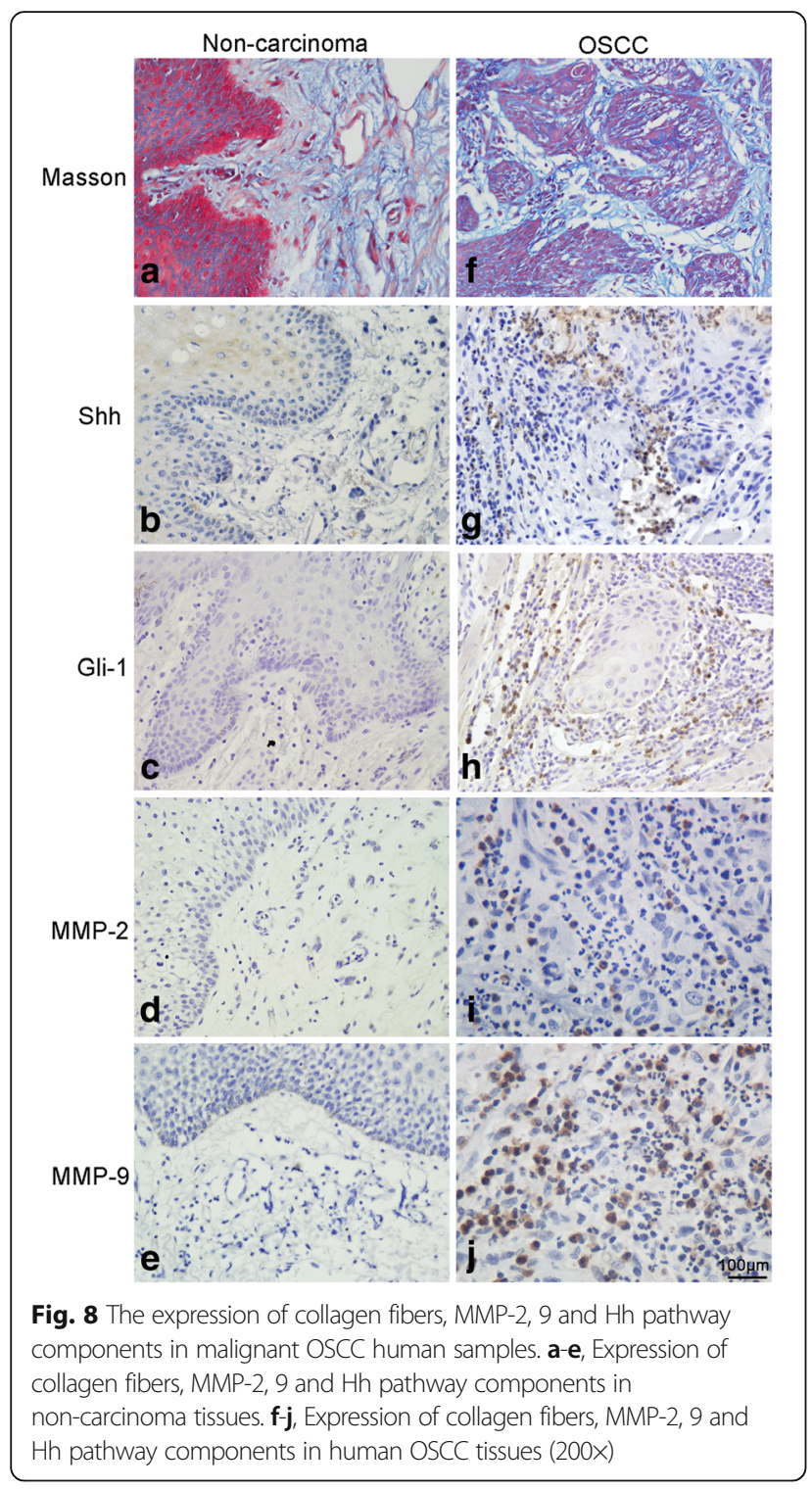

bodies induced by LIUS were also observed under TEM, which may be indicative of a cellular stress response (Fig. 1c). We demonstrated dynamic expression of the autophagy marker proteins LC3 and Beclin1 after ultrasound treatment, whereas the viabilities of cancer cells

Table 1 Association between expression of collagen fiber and MMP-2, MMP-9, Shh and Gli-1 in patients with OSCC by Spearman's correlation analysis

\begin{tabular}{lll}
\hline Molecule & \multicolumn{2}{l}{ Collagen fibers } \\
\cline { 2 - 3 } & Association coefficient (R) & $P$ value \\
\hline MMP-2 & -0.271 & $0.019^{\#}$ \\
MMP-9 & -0.459 & $0.000^{\#}$ \\
Shh & -0.351 & $0.002^{\#}$ \\
Gli-1 & -0.297 & $0.010^{\#}$ \\
\hline
\end{tabular}

$R$ Spearman's correlation coefficient

\# Statistically significant values $(p<0.05)$ were unaffected, possibly because autophagy functions as a protective response to LIUS, as demonstrated in previous studies $[21,28,29]$. We speculate that $1 \mathrm{~W} /$ $\mathrm{cm}^{2}$ LIUS causes "pores" on the cell surface, and that autophagy that is subsequently activated in response to stress protects the cells from injury [30, 31]. However, the ultrasound intensity used in this study does not produce sonoporation, so whether the "pores" on the cell surface induced by ultrasound rely on cell membrane disruption and/or caveolae-independent endocytosis needs further confirmation.

In order to verify the intracellular DOX concentration after $1 \mathrm{~W} / \mathrm{cm}^{2}$ LIUS treatment, we assessed the intracellular DOX levels by immunofluorescence. The results showed that after LIUS exposure, the cellular uptake of DOX increased, the activity was significantly inhibited, and apoptosis was increased. This occurred regardless of cell differentiation. Moreover, MTT assays showed no effect for normal HT293 cells treated under the same conditions. Thus, these results indicate that the increased in vitro cytotoxicity of DOX to human OSCC after ultrasound exposure is associated with increased intracellular DOX.

MMP-2 and MMP-9 are known to degrade the BM and ECM, and this degradation facilitates migration and invasion of tumor cells. Some studies suggest that the PI3K/AKT signaling pathway can promote tumor invasion and metastasis by controlling the expression of MMP-2 and MMP-9 [32]. Furthermore, combined LIUS and doxorubicin treatment may inhibit PI3K/Akt/NF- $\mathrm{KB}$ pathway activation [33]. Our results suggest LIUS and DOX combination treatment suppresses tumor growth, prolongs survival, increases the expression of MMP-2 and MMP-9, and promotes the deposition of collagen fibers in OSCC xenograft. These results indicate that a relative increase of collagen fibers can affect the invasion capacity of tumors. However, a previous study indicates that sufficient ultrasound intensity can damage interstitial collagen fibers, thereby reducing the compression of tumor vessels and causing increased blood flow and fast transfer of drugs to hypoxic areas, which increases the toxicity of chemotherapy [34]. In our experiments, no destruction of collagen fibers by LIUS was observed due to the use of different ultrasonic parameters or different tumor types.

As an upstream signaling activator of MMP-2 and MMP-9, the Hh signaling pathway plays an important role in the angiogenesis of adult mammalian animals and in cell migration $[35,36]$. In consideration of the Shh and Gli-1 results, we can speculate that the decreased MMP-2 and MMP-9 levels may be due to the inhibited activity of the Hh signaling pathway by high intracellular DOX concentrations, thus inhibiting tumor invasion and migration. The relationship between DOX 
and the Hh signaling pathway is definitely a worthwhile research topic for future investigation.

In addition to increased drug uptake, LIUS can enhance the sensitivity of drug-resistant cells, which provides a treatment strategy for multidrug resistance tumors [37]. Furthermore, DOX is an acoustic-sensitive agent that produces reactive oxygen and free radicals, which can further increase its cytotoxicity [38]. Future evaluation may clarify its mechanisms.

To validate the clinical significance of our results obtained from cell culture and animals, we collected clinical OSCC tissue samples from patients. We observed changes in the amount and morphology of collagen fibers, as well as increased expression of MMP-2, MMP-9, and their upstream regulators Shh and Gli-1, which provides a basis for targeting these signaling molecules. We speculate that this new model of adjuvant chemotherapy will allow the use of greatly reduced DOX doses in order to reduce systemic side effects. Therefore this regimen provides a promising new approach for the treatment of OSCC.

\section{Conclusion}

In summary, our in vitro and in vivo studies confirm that combination treatment with LIUS and DOX leads to the formation of temporary cell surface pores in OSCC cells, which in turn increases the cellular uptake of DOX. Combination treatment also down-regulates Hh signaling and decreases MMP-2 and MMP-9 expression. Furthermore, combination treatment leads to the aggregation of interstitial collagen fibers, which may inhibit tumor invasion to the surrounding tissues, resulting in prolonged survival times for tumor-bearing mice. As a non-invasive, targeted treatment, DOX and LIUS combination treatment overcomes the drawbacks of conventional clinical treatment modalities, therefore providing a useful approach for the treatment of OSCC.

\section{Additional file}

Additional file 1: Figure S1. Schematic diagram of the low-intensity ultrasound device and experimental setup. Figure S2. Schematic diagrams of ultrasound pressure level distribution for in vitro and in vivo experiments. Figure S3. The effects of LIUS and DOX on HT293 cell. (PPT $734 \mathrm{~kb}$ )

\section{Abbreviations \\ BM: Basement membrane; DOX: Doxorubicin; ECM: Extracellular matrix; Hh: Hedgehog; IHC: Immunohistochemical; LC3: Light chain 3; LIUS: Low intensity ultrasound; MMP-2: Matrix metalloproteinase-2; MMP-9: Matrix metalloproteinase-9; MMPs: Matrix metalloproteinases; OSCC: Oral squamous cell carcinoma; SEM: Scanning electron microscopy; Shh: Sonic hedgehog; TEM: Transmission electron microscopy; WB: Western blotting}

\section{Acknowledgements}

This work was supported by the National Natural Science Foundation of China (No.81502644 and No.81272503). The ultrasonic transducer used in this study was designed and manufactured by the Laboratory of Sono- and
Photo-theranostic Technologies of Harbin Institute of Technology. We would like to thank Letpub for providing editorial assistance.

\section{Authors' contributions \\ HXF and JHZ conceived and designed the experiments. HXF, HZ, GYL and $\mathrm{CW}$ performed the experiments and analyzed the data. HXL has made substantial contribution to collected tissue samples, and HXF, HXL and JHZ wrote the manuscript. WWC and $J \mathrm{HZ}$ reviewed the manuscript. All authors have read and approved the final manuscript.}

\section{Ethics approval and consent to participate}

All animal protocols were approved by the Laboratory Animal Committee of the Harbin Medical University. All patients gave informed consent, and the study was approved by the Research Ethics Committee of Harbin Medical University (Harbin, China).

\section{Competing interests}

The authors declare that they have no competing interests.

\section{Publisher's Note}

Springer Nature remains neutral with regard to jurisdictional claims in published maps and institutional affiliations.

\section{Author details}

'Departmentof Anatomy, Basic Medical Science College, Harbin Medical University, 194 Xuefu Road, Nangang District, Harbin 150081, China. ${ }^{2}$ Department of Oral Medicine, Jining Medical College, Shandong 272067, China. ${ }^{3}$ Department of Forensic Medicine, Basic Medical Science College, Harbin Medical University, Harbin 150081, China. ${ }^{4}$ Department of Oral Pathology, Stomatological Hospital, Harbin Medical University, Harbin 150001, China. ${ }^{5}$ Condensed Matter Science and Technology Institute, and Department of Physics, Harbin Institute of Technology, Harbin 150080, China. ${ }^{6}$ Materials Research Institute, The Pennsylvania State University, University Park, Pennsylvania 16802, USA.

Received: 13 August 2017 Accepted: 13 November 2017

Published online: 21 November 2017

\section{References}

1. Shah JP, Gil Z. Current concepts in management of oral cancer-surgery. Oral Oncol. 2009:45:394-401.

2. Mazeron R, Tao Y, Lusinchi A, Bourhis J. Current concepts of management in radiotherapy for head and neck squamous-cell cancer. Oral Oncol. 2009; 45:402-8.

3. Specenier PM, Vermorken JB. Current concepts for the management of head and neck cancer: chemotherapy. Oral Oncol. 2009;45:409-15.

4. Agulnik M. New approaches to EGFR inhibition for locally advanced or metastatic squamous cell carcinoma of the head and neck (SCCHN). Med Oncol. 2012;29:2481-91.

5. Quail DF, Joyce JA. Microenvironmental regulation of tumor progression and metastasis. Nat Med. 2013;19:1423-37.

6. Laitakari J, Stenback F. Collagen matrix in development and progression of experimentally induced respiratory neoplasms in the hamster. Toxicol Pathol. 2001;29:514-27.

7. Fan HX, Wang S, Zhao H, Liu N, Chen D, Sun M, et al. Sonic hedgehog signaling may promote invasion and metastasis of oral squamous cell carcinoma by activating MMP-9 and E-cadherin expression. Med Oncol. 2014;31:41.

8. Jain A, Rani V. Mode of treatment governs curcumin response on doxorubicin-induced toxicity in cardiomyoblasts. Mol Cell Biochem. 2017. doi:10.1007/s11010-017-3195-6.

9. Wen ZM, Jie J, Zhang Y, Liu H, Peng LP. A self-assembled polyjuglanin nanoparticle loaded with doxorubicin and anti-Kras siRNA for attenuating multidrug resistance in human lung cancer. Biochem Biophys Res Commun 2017:493:1430-7.

10. Zhu ZX, Sun CC, Ting Zhu Y, Wang Y, Wang T, Chi LS, et al. Hedgehog signaling contributes to basic fibroblast growth factor-regulated fibroblast migration. Exp Cell Res. 2017;355:83-94.

11. Zhou J, Zhu G, Huang J, Li L, Du Y, Gao Y, et al. Non-canonical GLI1/2 activation by PI3KNAKT signaling in renal cell carcinoma: a novel potential therapeutic target. Cancer Lett. 2016;370:313-23. 
12. Huaitong X, Yuanyong F, Yueqin T, Peng Z, Wei S, Kai S. Microvesicles releasing by oral cancer cells enhance endothelial cell angiogenesis via Shh/RhoA signaling pathway. Cancer Biol Ther. 2017;18(10):783-91.

13. Srinath S, lyengar AR, Mysorekar V. Sonic hedgehog in oral squamous cell carcinoma: an immunohistochemical study. J Oral Maxillofac Pathol. 2016; 20:377-83.

14. Yamanaka S, Tatsumi T, Shiraishi J, Mano A, Keira N, Matoba S, et al. Amlodipine inhibits doxorubicin-induced apoptosis in neonatal rat cardiac myocytes. J the Am Coll of Cardiol. 2003;41:870-8.

15. Riganti C, Voena C, Kopecka J, Corsetto PA, Montorfano G, Enrico E. Liposome-encapsulated doxorubicin reverses drug resistance by inhibiting P-glycoprotein in human cancer cells. Mol Pharm. 2011;8:683-700.

16. Primeau AJ, Rendon A, Hedley D, Lilge L, Tannock IF. The distribution of the anticancer drug doxorubicin in relation to blood vessels in solid tumors. Clin Cancer Res. 2005:11:8782-8.

17. Harrison GH, Balcer-Kubiczek EK, Gutierrez PL. In vitro action of continuouswave ultrasound combined with adriamycin, $X$ rays or hyperthermia. Radiat Res. 1996;145:98-101.

18. Saad AH, Hahn GM. Ultrasound-enhanced effects of adriamycin against murine tumors. Ultrasound Med Bio. 1992;18(8):715-23.

19. Rapoport N. Combined cancer therapy by micellar-encapsulated drug and ultrasound. Int J Pharm. 2004;277:155-62.

20. Park JH, Choi SH, Kim H, Ji ST, Jang WB, Kim JH, et al. Doxorubicin regulates Autophagy signals via accumulation of Cytosolic $\mathrm{Ca}_{2}{ }^{+}$in human cardiac progenitor cells. Int J Mol Sci. 2016;17(10):E1680.

21. Wang X, Wang P, Zhang K, Su X, Hou J, Liu Q. Initiation of autophagy and apoptosis by sonodynamic therapy in murine leukemia L1210 cells. Toxico in Vitro. 2013;27:1247-59.

22. Vonlaufen A, Joshi S, Qu C, Phillips PA, Xu Z, Parker NR, et al. Pancreatic stellate cells: partners in crime with pancreatic cancer cells. Cancer Res. 2008;68:2085-93.

23. Li H, Fan H, Wang Z, Zheng J, Cao W. Potentiation of scutellarin on human tongue carcinoma xenograft by low-intensity ultrasound. PLoS One. 2013;8: e59473.

24. Hu Z, LV G, Li Y, Li E, Li H, Zhou Q, et al. Enhancement of anti-tumor effects of 5-fluorouracil on hepatocellular carcinoma by low-intensity ultrasound. J Exp Clin Canc Res. 2016;35:71.

25. Fan HX, Li HX, Chen D, Gao ZX, Zheng JH. Changes in the expression of MMP2, MMP9, and Col IV in stromal cells in oral squamous tongue cell carcinoma: relationships and prognostic implications. J Exp Clin Canc Res. 2012;31:90.

26. Sumeth Perera MW, Gunasinghe D, Perera PA, Ranasinghe A, Amaratunga $P$, Warnakulasuriya $S$, et al. Development of an in vivo mouse model to study oral submucous fibrosis. J Oral Pathol Med. 2007;36:273-80.

27. Yu T, Wang Z, Mason TJ. A review of research into the uses of low level ultrasound in cancer therapy. Ultrason Sonoche. 2004;11:95-103.

28. Moretti L, Yang ES, Kim KW, Lu B. Autophagy signaling in cancer and its potential as novel target to improve anticancer therapy. Drug Resist Updat. 2007;10:135-43.

29. Zhang C, Jia X, Wang K, Bao J, Li P, Chen M, et al. Polyphyllin VII induces an Autophagic cell death by activation of the JNK pathway and inhibition of PI3K/AKT/mTOR pathway in HepG2 cells. PLoS One. 2016;11:e0147405.

30. Yu X, Luo A, Liu Y, Wang S, Li Y, Shi W, et al. MiR-214 increases the sensitivity of breast cancer cells to tamoxifen and fulvestrant through inhibition of autophagy. Mol Cancer. 2015;14:208.

31. Bursch W, Ellinger A, Kienzl $H$, Torok L, Pandey S, Sikorska M, et al. Active cell death induced by the anti-estrogens tamoxifen and ICI 164384 in human mammary carcinoma cells (MCF-7) in culture: the role of autophagy. Carcinogenesis. 1996:17:1595-607.

32. Chen J, Wang Q, Fu X, Huang X, Chen X, Cao L, Chen L, Tan H, Li W, Bi J, Zhang L. Involvement of PI3K/PTEN/AKT/mTOR pathway in invasion and metastasis in hepatocellular carcinoma: association with MMP-9. Hepatol Res. 2009;39:177-86.

33. Zhang Z, Xu K, Bi Y, Yu G, Wang S, Qi X, et al. Low intensity ultrasound promotes the sensitivity of rat brain glioma to doxorubicin by downregulating the expressions of $\mathrm{p}$-glucoprotein and multidrug resistance protein 1 in vitro and in vivo. PLoS One. 2013;8:e70685.

34. Li T, Wang YN, Khokhlova TD, D'Andrea S, Starr F, Chen H. Pulsed highintensity focused ultrasound enhances delivery of doxorubicin in a preclinical model of pancreatic cancer. Cancer Res. 2015;75:3738-46.
35. Suyama K, Onishi H, Imaizumi A, Shinkai K, Umebayashi M, Kubo M, et al. CD24 suppresses malignant phenotype by downregulation of $\mathrm{SHH}$ transcription through STAT1 inhibition in breast cancer cells. Cancer Lett. 2016;374:44-53.

36. Hooper JE, Scott MP. Communicating with hedgehogs. Nat Rev Mol Cell Bio. 2005;6:306-17.

37. Wang FZ, Xing L, Tang ZH, Lu JJ, Cui PF, Qiao JB, et al. Codelivery of doxorubicin and shAkt1 by poly (ethylenimine)-Glycyrrhetinic acid Nanoparticles to induce Autophagy-mediated liver cancer combination therapy. Mol Pharm. 2016;13:1298-307.

38. Tsai WB, Lai HY, Lee JL, Lo CW, Chen WS. Enhancement of the cytotoxicity and selectivity of doxorubicin to hepatoma cells by synergistic combination of galactose-decorated gamma-poly (glutamic acid) nanoparticles and lowintensity ultrasound. Langmuir. 2014;30(19):5510-7.

\section{Submit your next manuscript to BioMed Central and we will help you at every step:}

- We accept pre-submission inquiries

- Our selector tool helps you to find the most relevant journal

- We provide round the clock customer support

- Convenient online submission

- Thorough peer review

- Inclusion in PubMed and all major indexing services

- Maximum visibility for your research

Submit your manuscript at www.biomedcentral.com/submit
Biomed Central 